\title{
Design Low Profile and Wideband Antenna Based on Metasurface
}

\author{
Qahtan Mutar Gatea ${ }^{1}$, Abdulkadhum Jaafar Alyasiri ${ }^{1}$, Faris Mohammed Ali $^{1}$ and Nasr Al-Kafahji ${ }^{1}$ \\ ${ }^{1}$ Department of Communications Technical Engineering, Al-Najaf Technical Engineering College / Najaf, Al-Furat Al-Awsat \\ Technical University ATU, Najaf, 54001, Republic of Iraq \\ E-mail:qahtan9112@gmail.com; faris@atu.edu.iq;nasar@pdx.edu
}

\begin{abstract}
In this article, high gain, low-profile and wide bandwidth antenna, loaded with a single metasurface (MS) layer is proposed. The metasurface layer is composed of two sizes of periodic patches distributed along two dimensions. The size of large unit is divided to small four parts, establishing an approach wo operate with a wide range of frequencies. Therefore, the variations in the unit cell size assist to widen the proposed antenna bandwidth and matching enhancement as well. This metasurface layer is added above the slot antenna, separated by a distance $\left(h_{\text {air }}\right)$. The parameter $h_{\text {air }}$ plays a vital role in determining the overall performance. Although the whole antenna consists of two layers, it still has low profile of $0.115 \lambda 0$, where $\lambda 0$ is the wavelength at centre frequency of $6.2 \mathrm{GHz}$. The most important achievement in our proposed antenna that it has wideband characteristics for both gain and impedance bandwidth simultaneously. The impedance bandwidth value is $5.8 \%$ ranged from $6.15 \mathrm{GHz}$ to $6.52 \mathrm{GHz}$ for the slot antenna alone, while the slot antenna loaded with the metasurface layer has the value of $40 \%$ impedance bandwidth from $5.62 \mathrm{GHz}$ to $8.43 \mathrm{GHz}$. The peak gain attained in this research is $5.6 \mathrm{~dB}$, while it becomes 8.78 when adding the metasurface layer to the slot antenna. The gain enhancement is more than $3 \mathrm{~dB}$, considered as a good starting point to build antennas having high gains with no need to use antenna arrays which require special attention in their designs. Simulation results are as expected and provide a good comparison for the slot antenna before and after adding the metasurface to demonstrate the performance enhancement. The software used in all simulations is computer simulation technology CST.
\end{abstract}

Keywords - Metamaterial (MTM ); Metasurface ( MS ); Slot antenna ; Low profile; Wideband; High gain, Fabry- Pérot (F-P)

I. INTRODUCTION

Metasurfaces (MSs), which are twodimensional (2D) thin layers, consider as successor of metamaterials which are 3-dimensional structures. MSs are consisted of several unit cells arranged periodically along two dimensions, supported by a thin substrate. They ease the fabrication processes and reduce the losses introduced by their ancestors, metamaterials [1]. In the current decade, metasurfaces attract attention of a lot of researchers to address many problems confronted before with metamaterials, ranging from UHF band to terahertz applications. A plethora of promising applications such as flat lens, spatial filters, beam formers...etc find the metasurfaces good candidates to simply carry out these duties [1][2]. Therefore, MSs are considered as subdomains within the metamaterials [3]. Furthermore, the space between unit cells should not be larger than a quarter wavelength to guarantee that electromagnetic waves see homogenous materials.
The fascinating attributes of MSs lead to use them in broad range of applications such as compact planar circuits, polarization converter and rotator. In [4] [6], authors have used a diagonal metallic line in each center of unit cell, degenerating the incident electrical fields into two orthogonal modes with 90-degree phase shift. This process ensures the conversion of linear polarization waves to circular polarization. As known, the process of circular polarization generation requires two exciting sources, but MSs ease the process by using one exciting source. Another application where the MSs are used as surface-wave attenuator are widely utilized either in antenna arrays to reduce mutual coupling or in single antennas to improve the efficiency [7][8].

In both scenarios, these types of MSs called Electromagnetic band-gap structures EBGs resolve big problems which are the surface waves, known 
since the emergence of the first printed antennas. MSs, in other scenario called high impedance surfaces HISs, act to provide zero phase shift like magnetic conductors. The gap between, for example, the patch radiators in micro strip antennas and grounds are hugely shrunk, leading to low profile structures [9]. HISs, in literature, are also known as mushroom-like structures, reactive impedance surfaces RISs, or artificial magnetic conductors AMCs [10][11][12][13][14][15][16].

In this research scope, MSs are used to enhance the performance and conquer several drawbacks, confronted researchers when designing conventional antennas. To make the radiation pattern steerable, authors in [19], have reported a means where some active elements are integrated among unit cells, making their phase shifts dynamic. The variable phase shifts lead to change directions of radiation beams. $5 \mathrm{G}$ or bonyad designers find this technique very useful to use ideally an infinite number of small radiators (i.e., unit cells), able to shape the incoming waves as required without employing complicated circuits as in traditional phased arrays. In [20], the directivity has been enhanced by adding a metasurface layer above the antenna. To obtain linear to circular polarizer, low profile antenna, readers can refer to [17] and [18], respectively. Employing Huygens surfaces which are MSs to compensate phases deviations resulting from the out of phase radiations is reported in [21], whereas in [22], metalens based on the principles of optic transformation is introduced in compensate phases in plane-like radiations.

Applying MSs in a field of superstrates that have exceptional properties not available in standard ones is also explored, leading to obtain substrates not observed before. This exploration encourages a lot of researchers as in [23] to design an antenna with high gain, wideband, and low profile. Moreover, compact wideband directive metasurface CWMS, being a novel way to enhance the antenna directivity is presented in [24], where the excitation method used to feed the antenna is via twounequal slots. MS arrangement in a periodic fashion, composed of rectangular loop-based unit cells is deployed as a step to direct wave to the desired direction.

A leaky wave antenna based on MSs is proposed in [25]. The energy leaves the proposed structure gradually, wherever energy accumulated in space is added in phase, resulting in a constructive radiation pattern. Other works as in [26][27][28][29][30] are about using metasurfaces in antennas. Reference [26] suggests a structure consisting of frequent unit cells which are complementary split ring resonators CSRRs, being main radiators. There is no explicit antenna. The results obtained have showed that the proposed antenna has very wide bandwidth, starting from $5.64 \mathrm{GHz}$ to $5.8 \mathrm{GHz}$ with gain value of $5.8 \mathrm{~dB}$. Two interleaved hexagonal ring resonators based a single unit cell to create a meta surfaces layer is proposed in [27]. This makes the design complicated to some extent. Using double ring resonators has doubled the bandwidth more than $110 \%$ from $200 \mathrm{MHz}$ to $420 \mathrm{MHz}$ and the gain is increased to $7.88 \mathrm{~dB}$. Under the same reason explained above, authors in [28], introduces a meta surface layer having patch unit cells with different dimensions. The simulated results show a wide bandwidth from $4.2 \mathrm{GHz}$ to about $5.6 \mathrm{GHz}$ and gain values is equal to $8.2 \mathrm{~dB}$. placing the meta surface layer beneath the antenna is reported in [29]. This meta surface is contributed into the matching enhancement, resulting in wide bandwidth. The enhancement is more than 500\% where the bandwidth is increased from $100 \mathrm{MHz}$ to $630 \mathrm{MHz}$. In [30], meta material MTM which depends on a Sshaped unit cell in its structure has been proposed. This work considers one of works that introduces very wide bandwidth which is about $2 \mathrm{GHz}$ and average gain is equal to $5.53 \mathrm{~dB}$. the proposed antenna is linearly polarized slot antenna. Also, circular polarized meta surface antenna is proposed in [31]. The attained gain is about $7.1 \mathrm{~dB}$.

In our work, we propose a new design based on a bowtie-like slot antenna integrated with a meta surface layer fed by a micro strip transmission line, called a Fabry- Pérot FP structure. The whole structure can only support linear polarization waves. According to the best of authors' knowledge, our design introduces an antenna with really very wide band for the first time. It considers as a new contribution to the research field. The gain is also enhanced. Both increments in the bandwidth and gain can make our work distinct. The software used in all analysis is computer software technology CST.

Eventually, the paper is organized as follows: first, the slot antenna and meta surface are introduced in section I independently. Section II presents a detailed explanation about the adopted unit cell. In section III, the slot antenna integrated with the metasurface layer is given. All designs and simulations are clearly indicated. The last part, section IV, draws the conclusion.

\section{ANTENNA DESIGN}

This research mainly deals with the design of a small footprint antenna. To bear in mind, the proposed design size can be scaled up or down to 


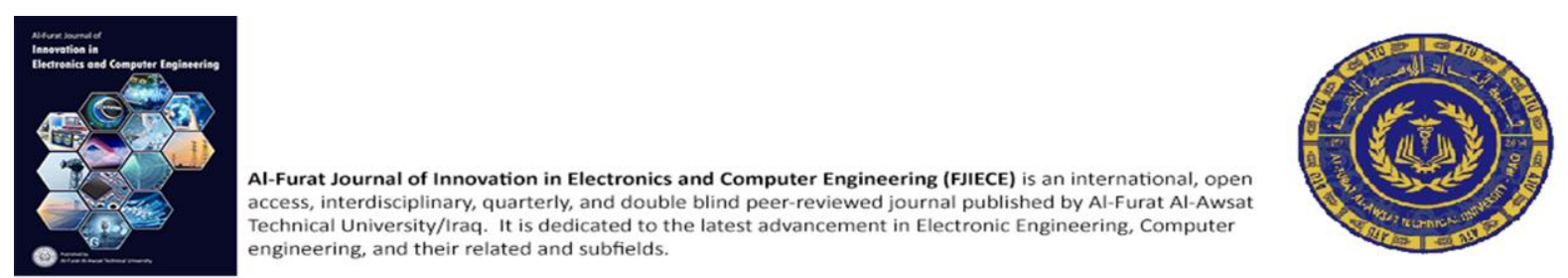

operate at any desired frequency, making an adjustable structure. In this section, we will discuss parts of the proposed antenna in detail, staring with the slot antenna as shown in Figure 1a. The slot antenna is cut out from a conductor layer placed over a RO4003C substrate with a dielectric constant 3.55 and a thickness of $1.5 \mathrm{~mm}$. The slot has two slot triangles attached to ends of slots, aiming to widen the bandwidth and enhancing the matching. This slot is excited by a micro strip feeding line with $50 \mathrm{ohm}$ characteristics impedance. This line extends about a quarter-wavelength at the desired frequency to excite the slot with a maximum current. The shape of extended line is a triangle as well for the same reason, broadening the bandwidth. Figure $1 \mathrm{~b}$ shows the metasurface layer used in the proposed structure. As can be seen, unit cells have two different sizes. Each four patches within the small size unit cells are equal to one size of the big unit cell.


(a)

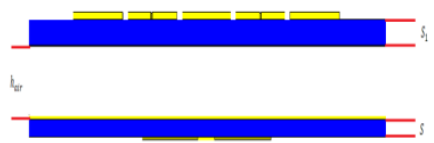

(c) (d)

(b)

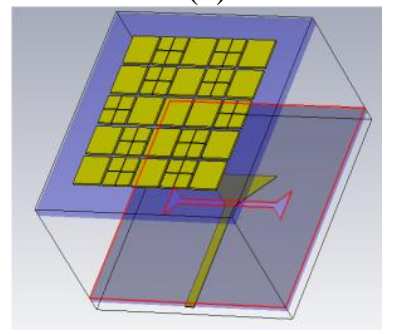

Figure.1: (a) Feed line and slot (b) Top view of metasurface (c) Side view of Antenna (d) 3D view of proposed Antenna

The variation in size results in a wide bandwidth. The distance separating between the slot antenna and the metasurface denoted by hair plays a vital role in determining the overall performance as will be seen later in this research study. Also, the

RO4003C substrate is used to design the metasurface layer. The thickness $S_{1}$ is $0.81 \mathrm{~mm}$, the loss tangent is 0.0025 , and the relative dielectric constant is 3.55 . The bigger unit cell has side length equal to $M_{X}=M_{y}=5.42 \mathrm{~mm}$ and the smaller one equal to $B_{x}=B_{y}=2.68 \mathrm{~mm}$, see Figure $1 \mathrm{~b}$. The air cavity, known as Fabry- Parot FP resonating cavity is optimized at $3 \mathrm{~mm}$ to obtain the highest possible gain. The radiated power is focused in smaller beams, resulting in high gain structures. For more information, interested readers can refer to [32]. The side view and three-dimensional views of the proposed antenna are portrayed in Figures $1 \mathrm{c}$ and 1d, respectively. Table I lists the proposed antenna dimensions. The dimensions given in the table are chosen after a huge number of attempts to optimize the antenna work.

Table I: Final antenna dimensions

$\begin{array}{cccc}\text { No } & \text { Parameters } & \text { Symbol } & \text { Value } \\ 1 & \text { The side length of big patch } & \text { Mx } & 5.42 \mathrm{~mm} \\ 2 & \text { The side width of big patch } & \text { My } & 5.42 \mathrm{~mm} \\ 3 & \text { The side length of small patch } & \text { Bx } & 2.68 \mathrm{~mm} \\ 4 & \text { The side width of small patch } & \text { By } & 2.68 \mathrm{~mm} \\ 5 & \text { Gap between cells } & \text { A } & 0.7 \mathrm{~mm} \\ 6 & \text { Gap between cells } & \text { C } & 0.16 \mathrm{~mm} \\ 7 & \text { Substrate length } & \text { Y } & 40 \mathrm{~mm} \\ 8 & \text { Substrate width } & \text { X } & 40 \mathrm{~mm} \\ 9 & \text { Length of feed line } & \text { Ry } & 26.5 \mathrm{~mm} \\ 10 & \text { Width of feed line } & \text { Rx } & 0.9 \mathrm{~mm} \\ 11 & \text { Slot length } & \text { Dx } & 12 \mathrm{~mm} \\ 12 & \text { Slot width } & \text { Dy } & 0.5 \mathrm{~mm} \\ 13 & \text { Air gap 1 } & \text { hair } & 3 \mathrm{~mm} \\ 14 & \text { Substrate of slot antenna } & \text { S } & 0.813 \mathrm{~mm} \\ 15 & \text { Substrate of metasurface } & \text { S1 } & 0.81 \mathrm{~mm}\end{array}$

The MS layer is added in the near field of the slot antenna, so the waves leaving the slot antenna that have spherical-like phase are converted to plane waves, being in phase. In other words, these periodic unit cells concentrate the EM waves in narrower volumetric space, leading to enhance an antenna gain and radiating for long distances. Furthermore, unit cells also improve an impedance bandwidth by adding many resonance effects into antenna response.

\section{RESULTS AND DISCUSSION}

Figure. 2 offers a comparison between results of only slot antenna and the slot integrated with metasurface layer. It is evidently observed how much bandwidth and gain have been attained when adding the metasurface layer. The bandwidth ranges from $6.15 \mathrm{GHz}$ to $6.52 \mathrm{GHz}$ for only slot antenna, being about $0.37 \mathrm{GHz}$. This narrowband behaviour is dramatically changed to very wideband about $2.81 \mathrm{GHz}$, extending from $5.62 \mathrm{GHz}$ to $8.43 \mathrm{GHz}$ once the metasurface layer is added above the slot antenna. 


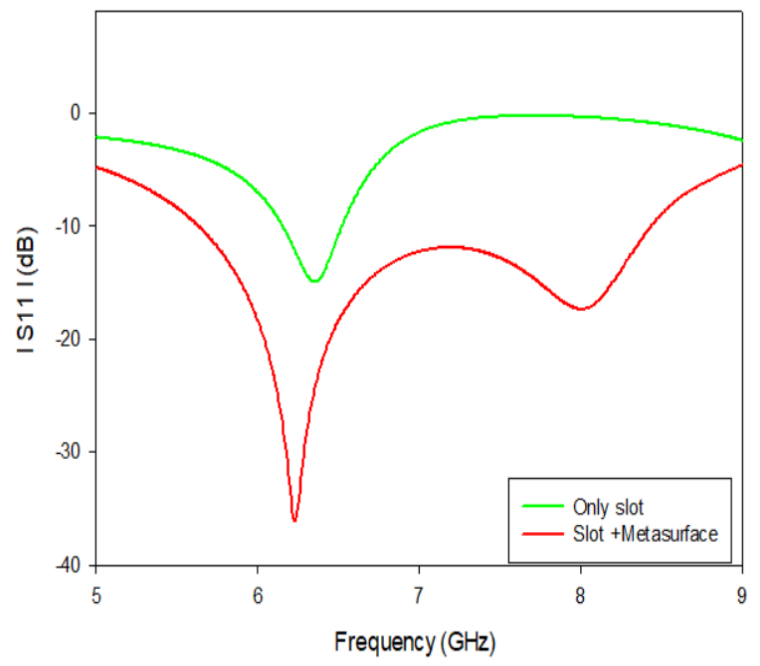

Fig. 2 : Reflection coefficient S11

As pointed out earlier, this procedure aids to enhance matching for more frequencies, making the antenna very wideband. The improvement in the antenna bandwidth makes it a potential candidate for be utilized in some commercial wireless applications. In the absence of the MS layer, the gain of the slot antenna is about $5.6 \mathrm{~dB}$, while in the presence of the metasurface layer, it results in $8.78 \mathrm{~dB}$ as a new gain as shown in Figure 3. The gain enhancement obtained by the proposed antenna was about $3.18 \mathrm{~dB}$.

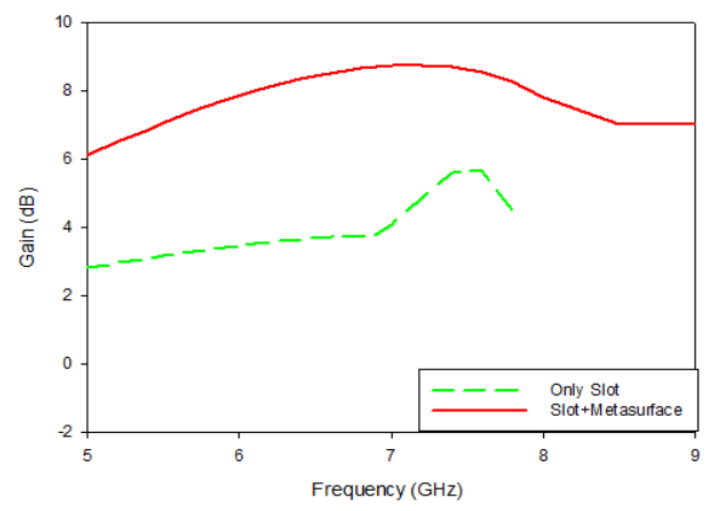

Fig.3 : Gain of slot antenna and metasurface

Moreover, the proposed antenna possesses gain values greater than a slot antenna over all frequencies. Increasing a gain value by $3.18 \mathrm{~dB}$ is not an easy task especially when utilizing small footprint single antennas where antenna designers know that. To do the same job using conventional ways, more than one antenna should be closely placed, called an antenna array with many disadvantages such as big footprints, matching complicated circuits, feeding network reequipment's, etc.
Next, after showing the gain and the bandwidth of the proposed antennas, it becomes imperative to show impacts of the air gap separating between the slot antenna and the metasurface layer on the bandwidth and gain of the proposed antenna, after several steps of optimization. Figures 4 and 5 display the reflection coefficient S11 bandwidth and the antenna gain. As can be seen, when the air gap is equal to $3 \mathrm{~mm}$, the antenna offers the widest bandwidth compared to other distances. However, this air gap does not provide the highest gain, but it is acceptable if we consider the bandwidth trading off. The one thing that should be mentioned here as the air gap distance increases, the antenna bandwidth deteriorates, belonging to capacitance impacts of the cavity, created by the substrate hosting the slot antenna and the metasurface layer. See the results when the air gap is equal to $3.5 \mathrm{~mm}$ and $4 \mathrm{~mm}$. As known the slot antenna has inductance characteristics, the capacitance provided by the cavity decreases for larger distances, thereby reducing the cancellation out of the slot antenna inductance. Thus, air gap with $3 \mathrm{~mm}$ is chosen.

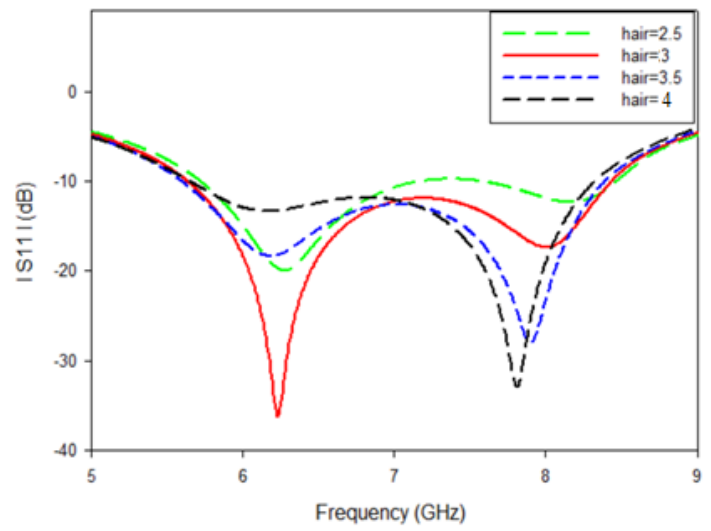

Fig.4 : Reflection coefficient S11 
Al-Furat Journal of Innovation in Electronics and Computer Engineering (FJIECE) is an international, open access, interdisciplinary, quarterly, and double blind peer-reviewed journal published by Al-Furat Al-Awsat Technical University/Iraq. It is dedicated to the latest advancement in Electronic Engineering, Computer engineering, and their related and subfields.

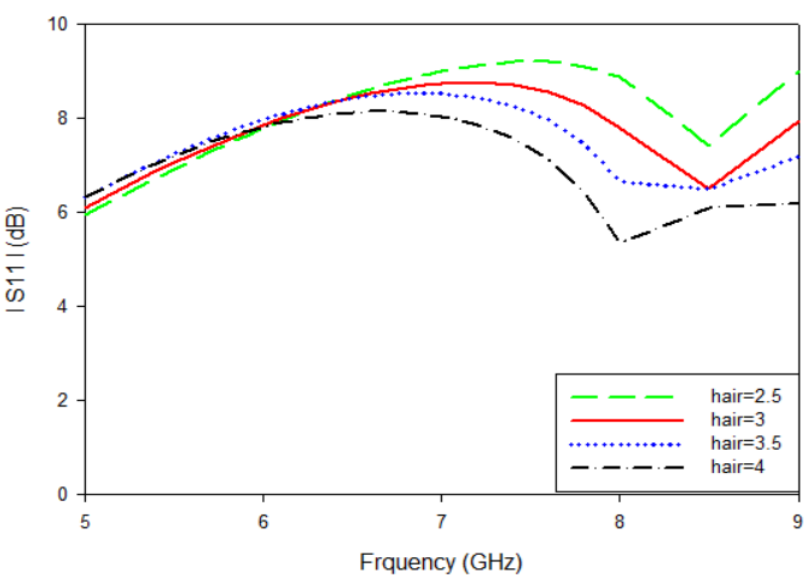

Fig.5 : Gain of proposed antenna

Table II introduces the results obtained for the slot antenna integrated with a metasurface layer, relying on the floquet theorem applied single unit cells of metasurfaces. This theorem provides whole information that the metasurface layer has negative permittivity, negative permeability, and negative refractive index using the $\mathrm{S}$ parameters algorithm. For more details, ref. [26] is the best option. The CST software was used in all analyses.

Table II : Proposed antenna result for (only slot antenna) and (slot antenna + metasurface layer)

$\begin{array}{cccc}\text { No } & \begin{array}{c}\text { Parameter } \\ \text { name }\end{array} & \text { Only slot antenna } & \begin{array}{c}\text { Slot antenna }+ \\ \text { metasurface }\end{array} \\ 1 & \begin{array}{c}\text { Resonant } \\ \text { Frequency } \\ 2\end{array} & 6.35 \mathrm{GHz} & 6.22 \mathrm{GHZ} \\ 3 & \text { Bandwidth } & \begin{array}{c}6.15 \mathrm{GHz}-6.52 \\ \mathrm{GHz}\end{array} & 5.62 \mathrm{GHz}-8.43 \mathrm{GHz} \\ 4 & \text { Return loss } & -14 \mathrm{~dB} & -36 \mathrm{dBi} \\ 5 & \text { Gain } & 5.6 \mathrm{~dB} & 8.78 \mathrm{dBi} \\ 6 & \begin{array}{c}\text { Directivity } \\ \text { Antenna } \\ \text { efficiency }\end{array} & 7.88 & 8.94 \mathrm{dBi} \\ & & 56 \% & 98 \%\end{array}$

Table III is very important because it shows the strength of our contribution through the exceptional increase in the bandwidth, gain, and matching level compared to other related work. The value of impedance bandwidth is $40 \%$, and this value can be obtained by applying Equation (2) in the reference [33]. This value is very excellent when compared to other reference, where work in [26] has value $2.7 \%$, $7.4 \%$ for [27], $28 \%$ for [28], $27 \%$ for [29] and $25 \%$ for [30].We also has increased the gain in the proposed antenna.
Table III : Comparing with other antennas

\begin{tabular}{|c|c|c|c|c|c|c|}
\hline No & Work & $\begin{array}{l}\text { Resonant } \\
\text { Frequency }\end{array}$ & Bandwidth & $\begin{array}{l}\text { Return } \\
\text { loss }\end{array}$ & Gain & Directivity \\
\hline 1 & [26] & $5.7 \mathrm{GHz}$ & $\begin{array}{c}5.64-5.8 \\
\mathrm{GHz}\end{array}$ & $\begin{array}{l}-27 \\
\mathrm{dBi}\end{array}$ & $\begin{array}{l}5.8 \\
\mathrm{dBi}\end{array}$ & - \\
\hline 2 & [27] & $5.7 \mathrm{GHz}$ & $\begin{array}{c}5.45-5.87 \\
\mathrm{GHz}\end{array}$ & $\begin{array}{l}-17 \\
\mathrm{dBi}\end{array}$ & $\begin{array}{l}7.88 \\
\mathrm{dBi}\end{array}$ & - \\
\hline 3 & [28] & $5 \mathrm{GHz}$ & $\begin{array}{c}4.20-5.59 \\
\mathrm{GHz}\end{array}$ & $\begin{array}{l}-21 \\
\mathrm{dBi}\end{array}$ & $\begin{array}{l}8.2 \\
\mathrm{dBi}\end{array}$ & - \\
\hline 4 & [29] & $2.3 \mathrm{GHz}$ & $\begin{array}{c}2-2.63 \\
\mathrm{GHz}\end{array}$ & $\begin{array}{l}-22 \\
\mathrm{dBi}\end{array}$ & $1 \mathrm{dBi}$ & - \\
\hline 5 & [30] & $7.6 \mathrm{GHz}$ & $7-9 \mathrm{GHz}$ & $\begin{array}{l}-32 \\
\mathrm{dBi}\end{array}$ & $\begin{array}{l}5.53 \\
\mathrm{dBi}\end{array}$ & \\
\hline 7 & work & $6.22 \mathrm{GHZ}$ & $\begin{array}{c}5.62-8.43 \\
\mathrm{GHz}\end{array}$ & $\begin{array}{l}-36 \\
\mathrm{dBi}\end{array}$ & $\begin{array}{c}8.78 \\
\mathrm{dBi}\end{array}$ & $\begin{array}{l}8.94 \\
\mathrm{dBi}\end{array}$ \\
\hline
\end{tabular}

\section{CONCLUSION}

A proposed antenna with many advantages such as high gain, low-profile and wideband was introduced in this research work. The metasurface used here to enhance the performance was periodic metallic patches with two different sizes. The idea behind that is to have a metasurface with wideband frequency response. The air gap has been optimized and its best value has been chosen as $3 \mathrm{~mm}$. This value has been selected to trade-off between the gain and impedance bandwidth. The metasurface was able to remove all inductance impacts of the slot antenna, enlarging the bandwidth. Moreover, the metasurface was able to focus the energy in narrower beams, leading to enhancing the antenna gain. $5 \times 5$ unit cells are used in all metasurfaces in this research work, the height profile is about $0.115 \lambda_{0}$ at the frequency of interest $6.22 \mathrm{GHz}$. Furthermore, the impedance bandwidth value is $5.8 \%$ ranging from $6.15 \mathrm{GHz}$ to $6.52 \mathrm{GHz}$ for only slot antenna, while the slot antenna loaded with the metasurface has the value of $40 \%$ starting from $5.62 \mathrm{GHz}$ to $8.43 \mathrm{GHz}$. The peak gain value is $5.6 \mathrm{~dB}$ for only slot antenna and $8.78 \mathrm{~dB}$ for the proposed antenna, slot antenna and metasurface layer, with gain enhancement about $3.18 \mathrm{~dB}$. Simulation results offer significantly improvement in the performance of the proposed antenna compared to the same without the metasurface layer. 


\section{REFERENCES}

[1] K. Konstantinidis, A. P. Feresidis, and P. Hall, "Broadband sub wavelength profile high-gain antennas based on multi-layer metasurfaces," IEEE Trans. Antenn. Propag. 63(1), 423-427 (2015)

[2] H. Li, G. Wang, H. X. Xu, T. Cai, and J. Liang, "X-Band phasegradient metasurface for high-gain lens antenna application,' IEEE Trans. Antenn. Propag. 63(11), 5144-5149 (2015).

[3] W. Cao, R. Singh, I. A. Al-Naib, M. He, A. J. Taylor, and W. Zhang, "Low-loss ultra-high-Q dark mode plasmonic Fano metamaterials," Opt. Lett. 37(16), 3366-3368 (2012).

[4] J. D. Ortiz, J. D. Baena, V. Losada, F. Medina, R. Marqués, and J. L. Araque Quijano, "Self-complementary metasurface for designing narrow band pass/stop filters," IEEE Microw. Wirel. Compon. Lett. 23(6), 291-293(2013).

[5] H. L. Zhu, S. W. Cheung, K. L. Chung, and T. I. Yuk, "Linear-tocircular polarization conversion using metasurface," IEEE Trans. Antenn. Propag. 61(9), 4615-4623 (2013).

[6] H. L. Zhu, X. H. Liu, S. W. Cheung, and T. I. Yuk, "Frequencyreconfigurable antenna using metasurface," IEEE Trans. Antenn. Propag. 62(1), 80-85 (2014).

[7] F. Yang and Y. Rahmat-Samii, "Reflection phase characterization of the EBG ground plane for low profile wire antenna applications," IEEE Trans. Antenn. Propag. 51(10), 2691-2703 (2003)

[8] N. Chamok, T. K. Anthony, S. J. Weiss, and M. Ali, "Ultra-thin UHF broadband antenna on a Non-Uniform Aperiodic (NUA) MetaSurface," IEEE Antennas Propag. Mag. 57(2), 167-180 (2015).

[9] C. R. Simovski, P. de Maagt, and I. V. Melchakova, "Highimpedance surfaces having stable resonance with respect to polarization and incidence angle," IEEE Trans. Antenn. Propag. 53(3), 908-914 (2005).

[10] A. Vallecchi, J. R. De Luis, F. Capolino, and F. De Flaviis, "Low profile fully planar folded dipole antenna on a high impedance surface," IEEE Trans. Antenn. Propag. 60(1), 51-62 (2012).

[11] S. X. Ta, I. Park, and R. W. Ziolkowski, "Circularly polarized crossed dipole on an HIS for 2.4/5.2/5.8-GHz WLAN applications," IEEE Antennas Wirel. Propag. Lett. 12(3), 1464-1467 (2013).

[12] S. N. Burokur, A.-C. Lepage, S. Varault, X. Begaud, G.-P. Piau, and A. de Lustrac, "Low-profile metamaterialbased L-band antennas," Appl. Phys., A Mater. Sci. Process. 122(4), 326-332 (2016).

[13] K. Agarwal, Nasimuddin, and A. Alphones, "Wideband circularly polarized AMC reflector backed aperture antenna," IEEE Trans. Antenn. Propag. 61(3), 1456-1461 (2013).

[14] B. S. Cook and A. Shamim, "Utilizing wideband AMC structures for high-gain inkjet-printed antennas on lossy paper substrate," IEEE Antennas Wirel. Prop. Lett. 12(1921), 76-79 (2013).

[15] P. Prakash, M. P. Abegaonkar, A. Basu, and S. K. Koul, "Gain enhancement of a CPW-Fed monopole antenna using polarizationinsensitive AMC structure," IEEE Antennas Wirel. Propag. Lett. 12(3), 1315-1318 (2013).

[16] S. X. Ta and I. Park, "Design of miniaturized dual-band artificial magnetic conductor with easy control of second/first resonant frequency ratio," Geochim. Cosmochim. Acta 13(2), 469-472 (2013)

[17] N. Nasimuddin, Z. N. Chen, and X. M. Qing, "Bandwidth enhancement of single-feed circularly polarized antenna using meta-surface," IEEE Antennas Propag. Mag. 16, 1045-9243 (2016).
[18] A. Monti, J. Soric, A. Alù, F. Bilotti, A. Toscano, and L. Vegni, "Overcoming mutual blockage between neighboring dipole antennas using a low-profile patterned metasurface," IEEE Antennas Wirel. Propag. Lett. 11(5), 1414-1417 (2012).

[19] T. I. Yuk, S. W. Cheung, and H. L. Zhu, "Mechanically pattern reconfigurable antenna using metasurface," Microwaves Antennas Prop. Lett. 9(12), 1331-1336 (2015).

[20] D. Blanco, E. Rajo-Iglesias, S. Maci, and N. Llombart, "Directivity enhancement and spurious radiation in leaky-wave antennas using inductive grid metasurfaces," IEEE Trans. Antenn. Propag. 63(3), 891 (2015).

[20] D. Blanco, E. Rajo-Iglesias, S. Maci, and N. Llombart, "Directivity enhancement and spurious radiation in leaky-wave antennas using inductive grid metasurfaces," IEEE Trans. Antenn. Propag. 63(3), 891 (2015).

[21] Chen, K., Yang, Z., Feng, Y., Zhu, B., Zhao, J., \& Jiang, T. (2015) Improving microwave antenna gain and bandwidth with phase compensation metasurface. AIP Advances, 5(6), 067152.

[22] Zhang, K., Ding, X., Wo, D., Meng, F., \& Wu, Q. (2016). Experimental validation of ultra-thin metalenses for $\mathrm{N}$-beam emissions based on transformation optics. Applied Physics Letters, 108(5), 053508

[23] Chaimool, S., Rakluea, C., \& Akkaraekthalin, P. (2011, December) Low-profile unidirectional microstrip-fed slot antenna using metasurface. In 2011 International Symposium on Intelligent Signal Processing and Communications Systems (ISPACS) (pp. 15). IEEE

[24] Majumder, B., Kandasamy, K., Mukherjee, J., \& Ray, K. P. (2015) Wideband compact directive metasurface enabled pair of slo antennas. Electronics Letters, 51(17), 1310-1312.

[25] Hosseini, A., Capolino, F., \& Jackson, D. R. (2015, July). Leakywave explanation of gain-bandwidth-enhanced Fabry-Perot cavity antennas formed by a thick multilayer partially-reflective surface. In 2015 IEEE International Symposium on Antennas and Propagation \& USNC/URSI National Radio Science Meeting (pp. 1090-1091). IEEE.

[26] A Rajak, N., \& Chattoraj, N. (2017). A bandwidth enhanced metasurface antenna for wireless applications. Microwave and Optical Technology Letters, 59(10), 2575-2580.

[27] Rajak, N., Chattoraj, N., \& Kumar, R. (2019). A gain and bandwidth enhanced metamaterial based surface antenna for wireles. communication. parameters, 50, 11

[28] Pan, Y. M., Hu, P. F., Zhang, X. Y., \& Zheng, S. Y. (2016). A low profile high-gain and wideband filtering antenna with metasurface. IEEE Transactions on Antennas and Propagation, 64(5), 2010-2016.

[29] Munir, A. (2017, October). Metasurface-backed monopole printed antenna with enhanced bandwidth. In 2017 International Symposium on Antennas and Propagation (ISAP) (pp. 1-2). IEEE.

[30] Rajesh, G. S., \& Kumar, V. (2015, December). Designing multiband metamaterial loaded microstrip patch antenna for SAR applications. In 2015 International Conference on Microwave and Photonics (ICMAP) (pp. 1-2). IEEE

[31] Zhou, C., Cheung, S. W., Li, Q., \& Li, M. (2017). Bandwidth and gain improvement of a crossed slot antenna with metasurface. Applied Physics Letters, 110(21), 211603.

[32] Wang, N., Liu, Q., Wu, C., Talbi, L., Zeng, Q., Xu, J.: “Wideband Fabry-Pérot resonator antenna with two complementary FSS layers', IEEE Transactions on Antennas and Propagation, 2014 62, (5), pp. 2463-2471

[33] Yang, Z. Z., Liang, F., Yi, Y., Zhao, D., \& Wang, B. Z. (2019) Metasurface-based wideband, low-profile, and high-gain antenna. IET Microwaves, Antennas \& Propagation, 13(4),436 441. 